122 COMPARING DIPEPTIDYL PEPTIDASE-4 INHIBITORS AND SODIUM-GLUCOSE COTRANSPORTER-2 INHIBITORS ON NEW-ONSET HEART FAILURE AND MYOCARDIAL INFARCTION

${ }^{1}$ Sharen Lee, ${ }^{2}$ Jiandong Zhou, ${ }^{2}$ Qingpeng Zhang, ${ }^{3}$ Gary Tse. ${ }^{1}$ Cardiovascular Analytics Group, Laboratory of Cardiovascular Physiology, Hong Kong, China; ${ }^{2}$ School of Data Science, City University of Hong Kong; ${ }^{3}$ Tianjin Institute of Cardiology, Second Hospital of Tianjin Medical University

\subsection{6/heartjnl-2021-BCS.119}

Introduction Sodium-glucose cotransporter-2 inhibitors (SGLT2I) and dipeptidyl-peptidase-4 inhibitors (DPP4I) are increasingly prescribed for type 2 diabetes mellitus patients. However, there are few population-based studies comparing their effects on incident heart failure (HF) or acute myocardial infarction (AMI). Therefore, the present study aims to compare the occurrence of major cardiovascular adverse events in SGLT2I and DPP4I users to evaluate their cardiovascular protective effects in a Chinese population.

Methods This was a population-based retrospective cohort study using the electronic health record database in Hong Kong, including type 2 diabetes mellitus patients receiving either SGLT2I or DPP4I between January 1st, 2015 to December 31st, 2020. Propensity-score matching was performed in a 1:2 ratio based on demographics, past comorbidities and medications using nearest-neighbor matching. Multivariate Cox regression analysis were used to identify significant predictors for new-onset HF or AMI, cardiovascular and all-cause mortality.

Results A total of 48875 and 49508 patients were included in the HF and AMI cohorts, respectively. After propensity score matching, SGLT2I use was associated with a lower risk of new-onset HF (HR: 0.41[0.27, 0.62], P<0.0001) and MI (HR: $0.52,95 \%$ CI: $[0.36,0.77], \mathrm{P}=0.0009$ ) in multivariable Cox models adjusted for demographics and past comorbidities compared to DPP4I use. SGLT2I users also had lower risks of all-cause and cardiovascular mortality $(\mathrm{HR}<1$, $\mathrm{P}<0.001)$.

Conclusions SGLT2 inhibitors are protective against adverse cardiovascular events including new-onset HF, MI, cardiovascular and all-cause mortality. The prescription of antidiabetic agents should be personalized, taking into consideration individual cardiovascular and metabolic risk profiles in addition to drug-drug interactions.

Conflict of Interest None

\section{CORONARY IMAGING OF CARDIAC ALLOGRAFT VASCULOPATHY PREDICTS CURRENT AND FUTURE DETERIORATION OF LEFT VENTRICULAR DYSFUNCTION IN PATIENTS WITH ORTHOTOPIC HEART TRANSPLANTATION}

${ }^{1}$ Ashwin Reddy, ${ }^{1}$ Bharat Khialani, ${ }^{1}$ Ben Lambert, ${ }^{1}$ Vincent Flore, ${ }^{1}$ Adam Brown, ${ }^{1}$ Nick West, 'Stephen Pettit, 'Jayan Parameshwar, ${ }^{1}$ Clive Lewis, 'Sai Bhagra, ${ }^{1}$ Anna Kydd, ${ }^{2}$ Stephen Hoole. ${ }^{1}$ Papworth Hospital, Cambridge, UK; ${ }^{2}$ Royal Papworth Hospital NHS Foundation Trust

\subsection{6/heartjn|-2021-BCS.120}

Background Cardiac allograft vasculopathy (CAV) is the leading cause of morbidity and mortality in heart transplant patients beyond the first post-transplant year, accounting for approximately $30 \%$ of all-cause mortality in this patient group. The incidence of CAV is $47 \%$ at 10 years post-transplant, though it can occur within the first year. Early detection of CAV is vitally important as modifications to medical therapy may slow progression of CAV and thus prevent downstream graft dysfunction. Intravascular Ultrasound (IVUS) and Optical Coherence Tomography (OCT) improve sensitivity of CAV detection compared to invasive coronary angiography (ICA), but the respective ability of each modality to predict downstream clinical events is unknown. Furthermore, whilst OCT has been shown to provide additional information about coronary vascular endothelial properties there is no consensus as to what constitutes 'severe' CAV on OCT, thus limiting its diagnostic utility. We sought to determine:1) The ability of OCT to diagnose CAV compared to IVUS, and determine a set cut-point for diagnosing 'severe' CAV on OCT.2) Whether severe CAV detected with ICA, IVUS or OCT correlates with future clinical events and graft function.

Methods Comparison of specific vessel parameters between IVUS and OCT on 20 patients attending for routine surveillance angiography 12-24 months post-orthotopic heart transplant. Major adverse cardiac events (MACE) and serial left ventricular ejection fraction were recorded prospectively. Comparisons of continuous data were performed using unpaired Student's t-tests and analysis of variance (ANOVA), whilst categorical data were compared using the $\chi 2$ test. A two-tailed probability level of $<0.05$ was considered significant.

Results Baseline demographic data for the 20 patients are shown in table 1 . Analyzing 55 coronary arteries we demonstrate that OCT and IVUS correlated well for vessel CAV characteristics (figure 1), although measured values were

Abstract 123 Table 1 Patient demographics and dinical characteristics. Data presented as mean \pm standard deviation or counts (percentage). $\mathrm{CCS}=$ Canadian Cardiovascular Society classification for angina, NYHA = New York Heart Association dassification for heart failure symptoms, NICMP= non-ischemic cardiomyopathy, $\mathrm{HCM}=$ hypertrophic cardiomyopathy, $\mathrm{AC}=$ arrhythmogenic cardiomyopathy, ICMP= ischaemic cardiomyopathy

\begin{tabular}{|l|l|}
\hline Variable & $\mathrm{N}=20$ \\
\hline Age/years & $46 \pm 14$ \\
\hline Male & $17(85)$ \\
\hline GFR/mI min ${ }^{-1} \mathbf{~ m}^{-2}$ & \\
$>60$ & $17(85)$ \\
$30-60$ & $3(15)$ \\
$<30$ & $0(0)$ \\
\hline CCS Classification & \\
CCS O & $20(100)$ \\
\hline NYHA Classification & \\
NYHA I & $16(80)$ \\
NYHA II & $4(20)$ \\
Donor Age/years & $36 \pm 14$ \\
Ischaemic time/minutes & $140.3 \pm 31.7$ \\
\hline Immunosuppression regime & \\
Tacrolimus - Mycophenolate & $3(15)$ \\
Sirolimus - Mycophenolate & $14(70)$ \\
Tacrolimus - Mycophenolate -Prednisolone & $2(10)$ \\
Sirolimus - Mycophenolate - Prednisolone & $1(5)$ \\
Primary diagnosis & $14(70)$ \\
NICMP & $3(15)$ \\
HCM & $2(10)$ \\
AC & $1(5)$ \\
ICMP & \\
\hline
\end{tabular}

